\title{
Optimal Biped Design Using a Moving Torso: Theory and Experiments
}

\author{
Karthik Kappaganthu ${ }^{1}$ and C. Nataraj ${ }^{2}$ \\ ${ }^{1}$ Principal Engineer, RED Global, Strategic Solution, LLC Virginia, VA 20165 \\ ${ }^{2}$ Professor \& Chair \\ Department of Mechanical Engineering, Villanova University, Villanova, PA 19085
}

USA

\section{Introduction}

Legged robots have been studied and developed for a long time. The primary advantage of legged motion is that they can traverse terrains inaccessible to wheeled robots. Biped robots are probably the most complicated of legged robots. Despite their complexity there has been a substantial amount of work done in the field including different techniques that have been developed to model, design and control biped robots. There are primarily two kinds of biped robots: active and passive bipeds. Many sophisticated, intelligent bipeds have been built by major companies. Most of the bipeds built are based on active control techniques; these are typically complicated, require high energies and are expensive to build. Also, they are far from mimicking true human motion. Owing to this, reliable biped robots are still elusive even to this day.

McGeer (1990) analyzed the natural dynamics of two-legged systems. He numerically and experimentally analyzed systems with concentrated masses on legs and hip. These bipeds did not have a torso and no external torques were applied. He showed that these systems could walk stably down small slopes and sustain the motion. This class of biped motion is known as Passive Dynamic Walking.

The step length and the velocity of the passive biped depend on parameters such as the masses, lengths and the slope on which the biped is walking. A passive biped is much more efficient than active bipeds and mimics the human motion better. Goswami, Espiau \& Keramane (1996); Goswami et al. (1993); Goswami, Thuilot \& Espiau (1996); Goswami, Thuilotz \& Espiauy (1996) describe the limit cycles and their stability in the passive gait of a biped without a torso. They obtained some numerical solutions of a system with known parameters. In Goswami et al. (1997) and Roussel et al. (98) bifurcation and chaos are studied and the dependence of the gait on the slope is explained.

Asano, Luo \& Yamakita (2004) use energy-based control laws to enable the biped to mimic the passive motion; they analyze a biped without torso and knees. Asano, Yamakita, Kamamichi \& Luo (2004), Kim \& Oh (2004), Paul et al. (2003), Silva \& Machado (2001) and Goswami (1999) are some more of the innovative attempts to build a simple yet controllable walking machine. Although passive dynamic walking is efficient, simple to implement and analyze, the step length and the velocity of motion of the passive biped are greatly dependent on the system parameters. The path followed by the biped is uncontrollable and there is no control 


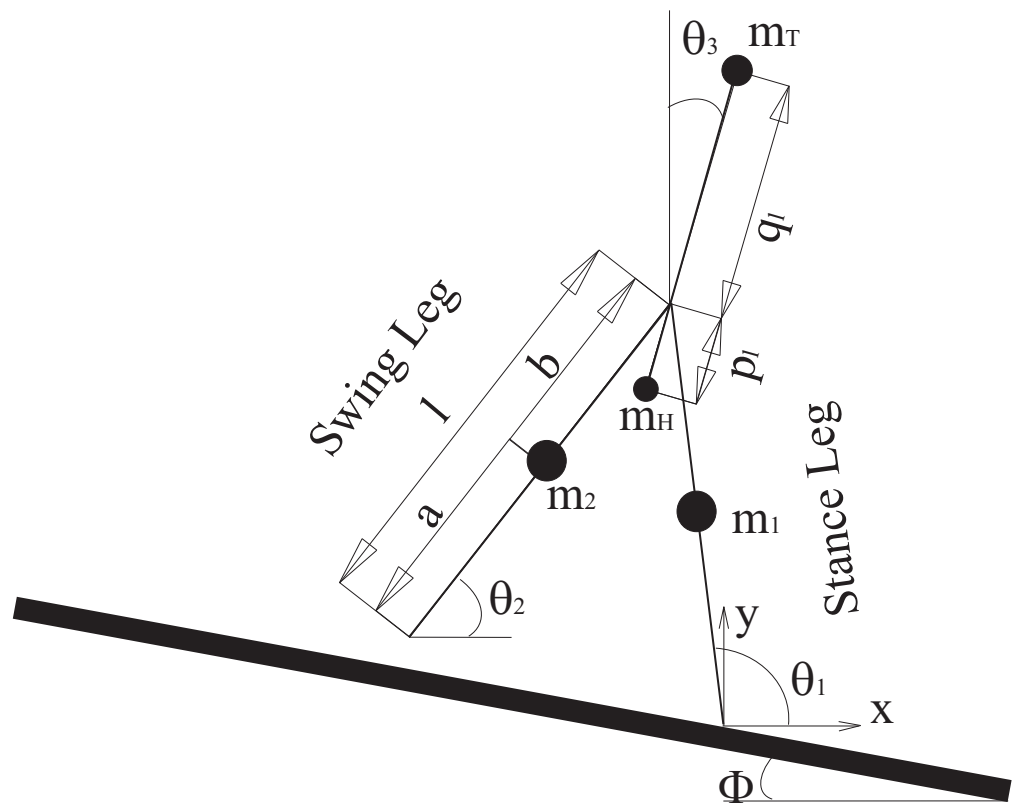

Fig. 1. Two Dimensional Model of the Biped

whatsoever on the system performance. This makes passive bipeds unrealistic for practical applications.

Actively controlled bipeds on the other hand are very adaptable to path planning and stability control at the cost of simplicity. Further they are typically prohibitively costly. Recognizing this, some attempts have been made to incorporate passive dynamics into actively controlled bipeds as it could theoretically result in an efficient method.

The torso plays an important role in the motion of bipeds. Typically most of the mass is concentrated on the torso or on the hip. It can be observed even in human motion that the position of the torso changes with a change in slope. Athletes also seem to use the torso to gain efficiency. Our present work is motivated by this influence the torso has on the biped. Although many bipeds with torsos have been built, little work has been done to utilize the effect of the torso on dynamics. This work tries to incorporate torso dynamics into the control of bipeds.

This paper analyzes the effect of the torso on the step length and velocity of a biped walking down a known slope. This information is used to control the step length and velocity of biped by applying the torque solely at the hip. The legs are passive at all times. The motion of legs is controlled solely by the mathematical coupling between the motion of the torso and the legs. The biped analyzed is shown in Fig. 1.

The goal of this work is to control just the step-length and velocities; clearly, there are infinitely many path profiles that can be executed to achieve this goal. Each path profile has a corresponding torque and external energy associated with it. In this work the path profile that minimizes the external energy input is found and the corresponding torque is applied. A biped is built to implement and test the effect of torso on the biped motion. The biped walks only down the slopes. The legs are not actuated externally. Different torques are input to the 
biped to make it walk with a required step length. The biped weighs $7 \mathrm{~kg}$ and is $60 \mathrm{~cm}$ tall. A DC motor is used to actuate the torso.

The second section provides the mathematical model for the biped being considered. It explains the phases of biped motion and derives the governing equation of motion. A brief introduction to terminology in bipeds and computational methods in passive bipeds is also provided. The third section presents the simulations of the biped in forward and inverse dynamics. Trajectories for typical parameter values are solved for and the variation of potential energy and kinetic energy is studied. Also the dependence of biped motion on the torso is studied. Section Four defines the optimal problem and its corresponding solution. Optimal trajectories and torques are obtained. Section Five explains the experimental setup, and Section Six, the results of practical application of the principle. Finally, Section Seven summarizes the work and presents a list of future tasks to be accomplished.

\section{Modeling}

The biped is modeled in two dimensional space. It has two legs without knees, and the mass is assumed to be concentrated at the center of mass of each leg. The lengths of both legs are equal and the two legs are connected by a hip. There is a third mass on the torso which is at the center of the hip. The motor mass is assumed to be concentrated at a known distance from the hip. The degrees of freedom and parameters are as shown in Fig. 1.

The basic motion consists of two phases: the swing phase and the impact phase. The swing phase consists of motion of the swing leg, and the motion is described by a continuous differential equation obtained from Lagrange equations. The impact phase consists of instantaneous impact of the swing leg and the transition to the next step. The following assumptions are made in deriving the equations of motion.

- The biped is modeled only in the lateral plane; the motion in the longitudinal plane is neglected.

- The impact of the swing leg at the end of each step is infinitesimally small.

- The impact of the swing leg is plastic.

- After the impact phase the functionality of each leg is interchanged; i.e., the swing leg becomes the stance leg, and vice versa.

- The angular momentum is conserved during impact.

With these assumptions, the governing equation of the biped can be derived for the swing phase as follows.

$$
M(q, \dot{q}) \ddot{q}+C(q, \dot{q})+K(q)=F, \text { when } \theta_{1}+\theta_{2} \neq \pi-2 \phi
$$

The impact occurs when $\theta_{1}+\theta_{2}=\pi-2 \phi$; the states after impact are given by

$$
\begin{aligned}
q^{+} & =\left(\begin{array}{lll}
0 & 1 & 0 \\
1 & 0 & 0 \\
0 & 0 & 1
\end{array}\right) q^{-} \\
\dot{q}^{+} & =\left(\begin{array}{lll}
0 & 1 & 0 \\
1 & 0 & 0 \\
0 & 0 & 1
\end{array}\right)\left\{q^{-}+\left[J M^{-1} J^{T}\right]^{-1} \delta \dot{r_{e}}\right\}
\end{aligned}
$$


$r_{e}$ is a vector containing the co-ordinates of the end-point of the swing leg. $M, C, K, F$, and $J$ are system matrices as described by Eqs. 4-8.

$$
\begin{aligned}
& M(q)= \\
& {\left[\begin{array}{ccc}
m_{1} a^{2}+\left(m_{T}+m_{H}+m_{2}\right) \ell^{2} & -m_{2} b \ell \cos \left(\theta_{1}-\theta_{2}\right) & \left(m_{H} p_{l}-m_{T} q_{l}\right) \ell \sin \left(\theta_{1}+\theta_{C}\right) \\
-m_{2} b \ell \cos \left(\theta_{1}-\theta_{2}\right) & m_{2} b^{2} & 0 \\
\left(m_{H} p_{l}-m_{T} q_{l}\right) \ell \sin \left(\theta_{1}+\theta_{C}\right) & 0 & m_{H} p_{l}^{2}+m_{T} q_{l}^{2}
\end{array}\right]} \\
& C(\dot{q}, q)=\left[\begin{array}{c}
\left(m_{H} p_{l}-m_{T} q_{l}\right) \ell \cos \left(\theta_{1}-\theta_{2}\right) \dot{\theta}_{1}^{2}-m_{2} b \ell \sin \left(\theta_{1}-\theta_{2}\right) \dot{\theta}_{2}^{2} \\
m_{2} b \ell \sin \left(\theta_{1}-\theta_{2}\right) \dot{\theta}_{1}^{2} \\
\left(m_{H} p_{l}-m_{T} q_{l}\right) \ell \cos \left(\theta_{1}+\theta_{C}\right) \dot{\theta}_{1}^{2}
\end{array}\right] \\
& K(q)=\left[\begin{array}{c}
{\left[m_{1} a+\left(m_{H}+m_{T}+m_{2}\right) \ell\right] g \cos \theta_{1}} \\
-m_{2} g b \cos \theta_{2} \\
\left(m_{H} p_{l}-m_{T} q_{l}\right) g \sin \theta_{C}
\end{array}\right] \\
& J=\frac{\partial \mathrm{r}_{\mathrm{e}}}{\partial \mathrm{q}} \\
& F=\left[\begin{array}{l}
0 \\
0 \\
\tau
\end{array}\right]
\end{aligned}
$$

The parameters of motion are the step length $(\lambda)$ and the time taken for each step $\left(t_{\text {end }}\right)$; $t_{\text {end }}$ can be obtained by solving

$$
\theta_{1}\left(t_{\text {end }}\right)+\theta_{2}\left(t_{\text {end }}\right)=\pi-2 \phi
$$

The step length $\lambda$ is then given by

$$
\lambda=\sqrt{\left(x_{e f}-x_{e i}\right)^{2}+\left(y_{e f}-y_{e i}\right)^{2}}
$$

where, $x_{e}, y_{e}$, the co-ordinates of the end point of the swing leg, are given by

$$
\begin{aligned}
& x_{e}=\ell \cos \theta_{1}-\ell \cos \theta_{2} \\
& y_{e}=\ell \sin \theta_{1}-\ell \sin \theta_{2}
\end{aligned}
$$

$x_{e i}, y_{e i}$ are the co-ordinates at $t=0$, and, $x_{e f}, y_{e f}$ are the co-ordinates at $t=t_{\text {end }}$.

\section{Simulation}

There are two stages of simulation: forward dynamics and inverse dynamics. Forward dynamics refers to the solution of the equations to compute the dynamic response to the imposed excitations, and is typically done using a standard procedure such as the Runge Kutta algorithm (which is the one we used). Inverse dynamics is the computation of the excitation necessary to achieve a certain prescribed motion, and is described in the next subsection.

The initial choice of parameters is such that they are close to the physical system. Later some parameters are varied so that the dependence of the path on the parameters can be verified. The parameter values are chosen considering the final biped to be built and also care is taken that the parameters do not create singularities in the problem. The parameter values chosen are given in Table 1. 


\begin{tabular}{|l|c|}
\hline \multicolumn{2}{|c|}{ Parameters } \\
\hline Parameter & Value \\
\hline$m_{1}$ & $0.5 \mathrm{Kg}$ \\
$m_{H}$ & $2.5 \mathrm{Kg}$ \\
$m_{T}$ & $3.5 \mathrm{Kg}$ \\
$m_{2}$ & $0.5 \mathrm{Kg}$ \\
$\mathrm{a}$ & $0.14 \mathrm{~m}$ \\
$\mathrm{~b}$ & $0.21 \mathrm{~m}$ \\
$p_{l}$ & $0.1 \mathrm{~m}$ \\
$q_{l}$ & $0.2 \mathrm{~m}$ \\
$\ell$ & $0.35 \mathrm{~m}$ \\
$\phi$ & $3^{\mathrm{o}}$ \\
\hline
\end{tabular}

Table 1. Parameter Values

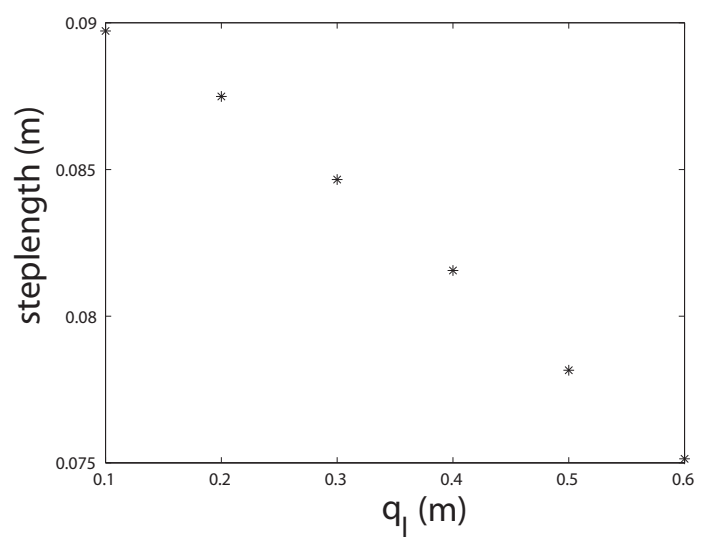

Fig. 2. Dependence of step length on $q_{l}$

To check for the dependence of the trajectories on the torso the parameters corresponding to the torso, $p_{l}$ and $q_{l}$, are varied; the torso parameters appearing as moment terms $m_{T}, m_{H}$ are held constant.

The results of the forward dynamic simulation are as follows. Mass $m_{T}$ has a decreasing effect on $\lambda$ and $t_{\text {end }}$. As shown in Fig. $2, \lambda$ decreases from $9 \mathrm{~cm}$ to $7.5 \mathrm{~cm}$ when $q_{l}$ changes from 0.1 to $0.6 \mathrm{~cm}$. As expected the decrease in $\lambda$ causes a decrease in $t_{\text {end }}$ from $0.22 \mathrm{sec}$ to $0.16 \mathrm{sec}$, as shown in Fig. 3. It can be seen from the graphs that the change in the $t_{\text {end }}$ is higher near smaller values of $q_{l}$.

Mass $m_{H}$ has an increasing effect on $\lambda$ and $t_{\text {end }}$. $\lambda$ increases from $6.6 \mathrm{~cm}$ to $9 \mathrm{~cm}$ when $p_{l}$ changes from 0.1 to $0.6 \mathrm{~cm}$. As expected the increase in $\lambda$ causes an increase in $t_{\text {end }}$ from 0.15 sec to $0.23 \mathrm{sec}$. These are shown in Figs. 4 and 5 respectively.

In order to visualize the change in the shape of the profile, position vector of end point of the swing leg $\left(x_{e}, y_{e}\right)$ is plotted qualitatively for changes in $q_{l}$ and $p_{l}$. These are shown in Figs. 6 and 7 respectively. In each of the plots the number at the end of the arrow denotes the value of $q_{l}$ (or $p_{l}$ ) at which the profile was generated. The dotted line represents the slope on which the biped is walking. 


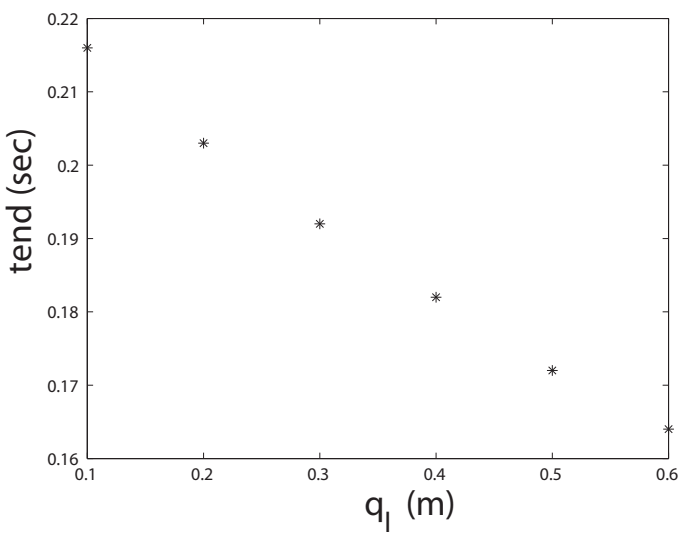

Fig. 3. Dependence of step-end time on $q_{l}$

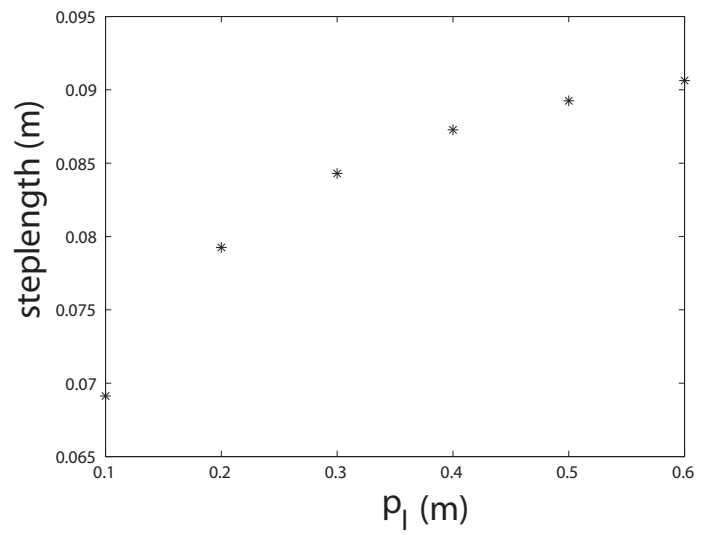

Fig. 4. Dependence of step length on $p_{l}$

\subsection{Inverse Dynamics}

In order to make the biped move with a predetermined step length and velocity, an external torque needs to be applied. The external torque is applied only at the torso. In order to determine the torque we need to solve the inverse dynamics problem. The flowchart to determine the torque by inverse dynamics is shown in Fig. 8.

Figure 9 is the input and Fig. 10 is the output of the algorithm. Note that $\theta_{1}$ is monotonic in this case. The shape of the feet profile is shown in Fig. 11. The step length and step-end time chosen are $6 \mathrm{~cm}$ and $0.43 \mathrm{sec}$ respectively. The maximum torque applied is $4.75 \mathrm{Nm}$.

\section{Optimization}

The choice of the profile of $\theta_{1}$ has been arbitrary in the inverse dynamics problem. Now, we focus on the development of an optimal trajectory for the biped. The objective here is to 


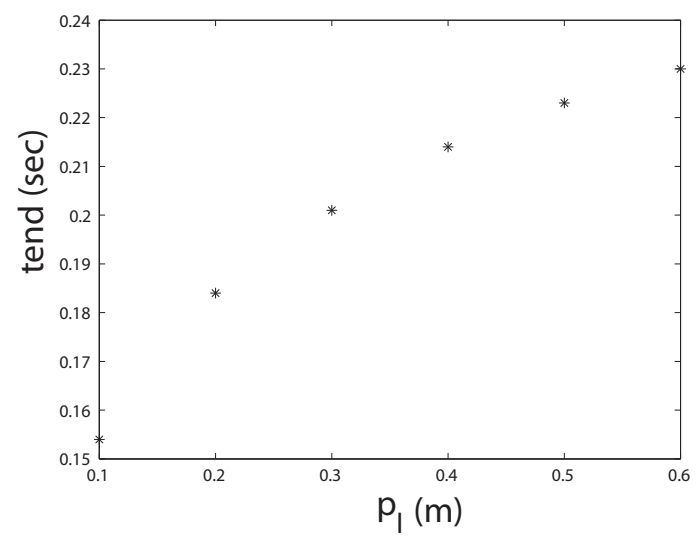

Fig. 5. Dependence of step-end time on $q_{l}$

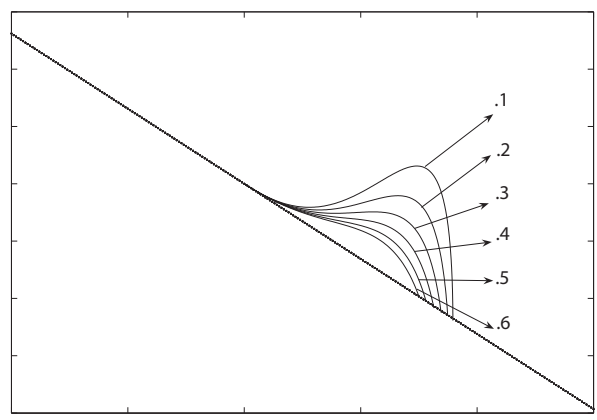

Fig. 6. Qualitative shape of the feet profile for varying $q_{l}$ as numbered (see text for explanation)

minimize the external energy input to the system given the governing dynamics, step length and the time-period.

The external energy is supplied in the form of a torque which is applied at the torso. Eq. 20 gives the total external energy supplied to the biped. In the optimal problem the profiles of $\theta_{1}$, $\theta_{2}$ are reasonably fitted to a cubic curve as shown in Eqs. 13 and 14. There are four unknown coefficients for each variable. Since the initial conditions, $\lambda$ and $t_{\text {end }}$ are known and since the profiles should satisfy these conditions, the number of independent coefficients for each profile is two. These coefficients are the design variables for the optimization problem.

Since the profiles of both $\theta_{1}$ and $\theta_{2}$ are chosen they should satisfy the governing equation, hence the second equation in the governing equation that couples $\theta_{1}$ and $\theta_{2}$ becomes the nonlinear equality constraint for the optimization shown in Eq. 22. Also, it should be taken care that the feet-profile is consistent with the slope; i.e., during the swing phase it should always be above the ground. This can be modeled using geometry by ensuring that each point of the feet profile when substituted in the equation of the slope should be non-negative. 


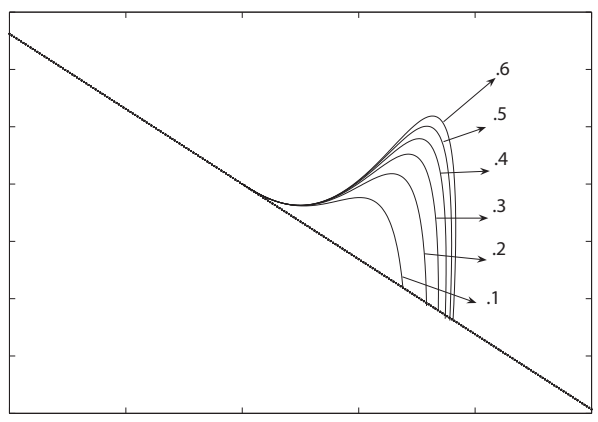

Fig. 7. Qualitative shape of the feet profile for varying $p_{l}$ as numbered (see text for explanation)

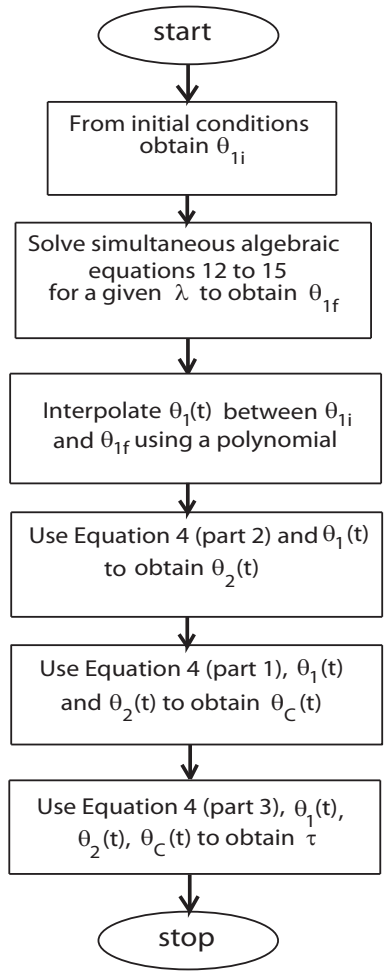

Fig. 8. Flowchart for inverse dynamics $(\lambda)$ 


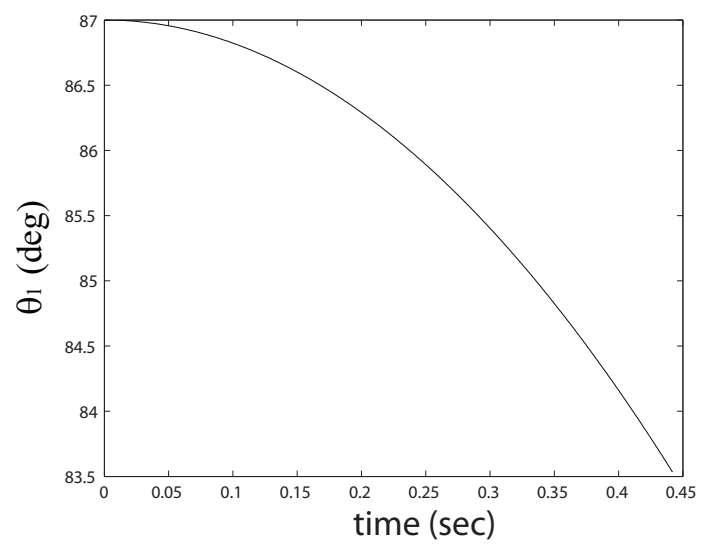

Fig. 9. $\theta_{1}^{\text {inv }}$ Obtained for the chosen step length by interpolation

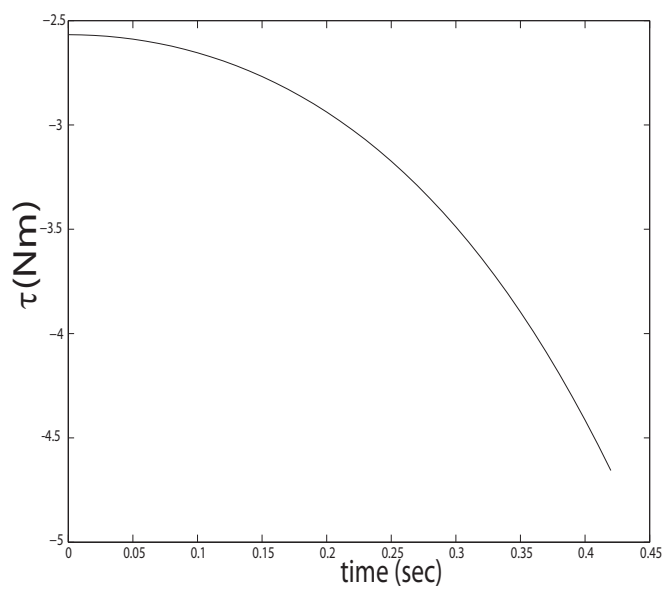

Fig. 10. Torque obtained for the chosen step length from inverse dynamics

This becomes the nonlinear inequality constraint shown in Eq. 23. The optimal problem formulation is shown in the next subsection.

\subsubsection{Problem definition}

Let

$$
\begin{aligned}
& \theta_{1_{\mathrm{opt}}}(t)=a_{0}+a_{1} t+a_{2} t^{2}+a_{3} t^{3} \\
& \theta_{2_{\mathrm{opt}}}(t)=b_{0}+b_{1} t+b_{2} t^{2}+b_{3} t^{3}
\end{aligned}
$$




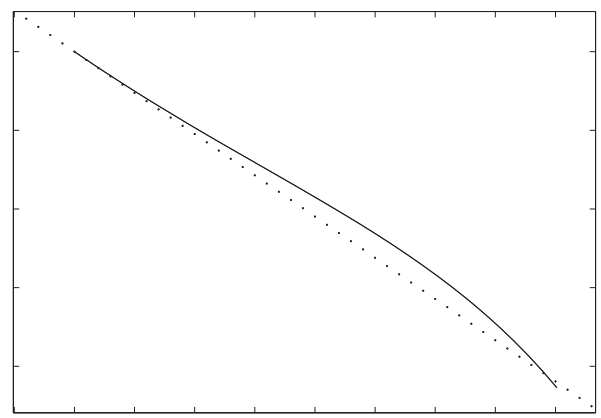

Fig. 11. Qualitative shape of feet profile for the chosen step length from inverse dynamics

Since $\theta_{1_{\mathrm{opt}}}$ and $\theta_{2_{\mathrm{opt}}}$ satisfy the initial and end condition

$$
\begin{aligned}
& a_{0}=\theta_{1_{i}} \\
& b_{0}=\theta_{2_{i}} \\
& a_{1}=\frac{\theta_{1_{f}}-\theta_{1_{i}}-a_{2} t_{\mathrm{end}}^{2}-a_{3} t_{\mathrm{end}}^{3}}{t_{\mathrm{end}}} \\
& b_{1}=\frac{\theta_{2_{f}}-\theta_{2_{i}}-b_{2} t_{\mathrm{end}}^{2}-a_{b} t_{\mathrm{end}}^{3}}{t_{\mathrm{end}}}
\end{aligned}
$$

The design variables for the optimization problem are defined as

$$
x_{\mathrm{opt}}=\left[\begin{array}{l}
a_{3} \\
a_{2} \\
b_{3} \\
b_{2}
\end{array}\right]
$$

The total external energy supplied per step is

$$
E_{\text {ext }}\left(x_{\mathrm{opt}}\right)=\int_{t_{0}}^{t_{\text {end }}} \tau \dot{\theta_{C}} d t
$$

The optimal problem is hence (minimization of energy):

$$
\operatorname{Min} E_{\text {ext }}\left(x_{\mathrm{opt}}\right)
$$

such that

$$
\begin{aligned}
-m_{2} \ell b \cos \left(\theta_{1_{\mathrm{opt}}}-\theta_{2_{\mathrm{opt}}}\right) \ddot{\theta}_{1_{\mathrm{opt}}}+m_{2} b^{2} \ddot{\theta}_{1_{\mathrm{opt}}}+m_{2} \ell b \sin \left(\theta_{1_{\mathrm{opt}}}-\theta_{2_{\mathrm{opt}}}\right) \dot{\theta}_{1_{\mathrm{opt}}}^{2} & \\
-m_{2} g b \cos \theta_{2_{\mathrm{opt}}} & =0 \\
-y_{e}-\tan \phi x_{e} & \leq 0
\end{aligned}
$$

where, $y_{e}$ and $x_{e}$ are the co-ordinates of the end point of the swing leg. Sequential Quadratic Programming is used to solve the optimal problem. Solving the optimal problem with $\lambda=6$ $\mathrm{cm}$ and $t_{\mathrm{end}}=0.35$, the maximum torque obtained here is less than half that obtained by 


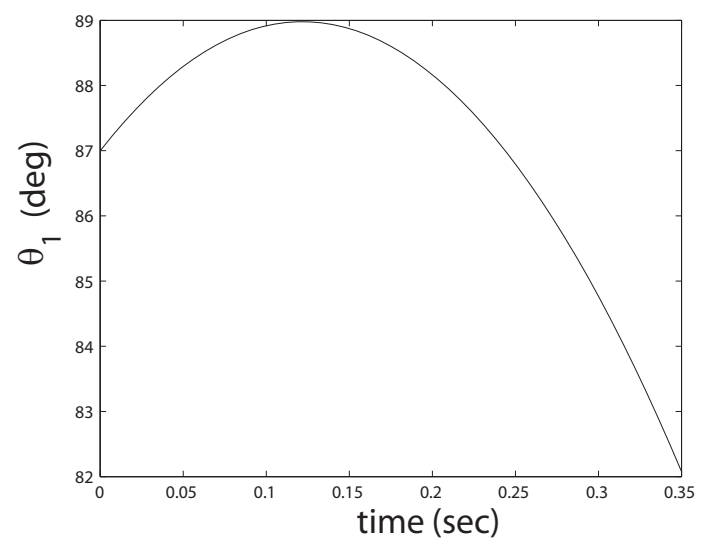

Fig. 12. Optimal trajectory for $\theta_{1}$ for the chosen step length

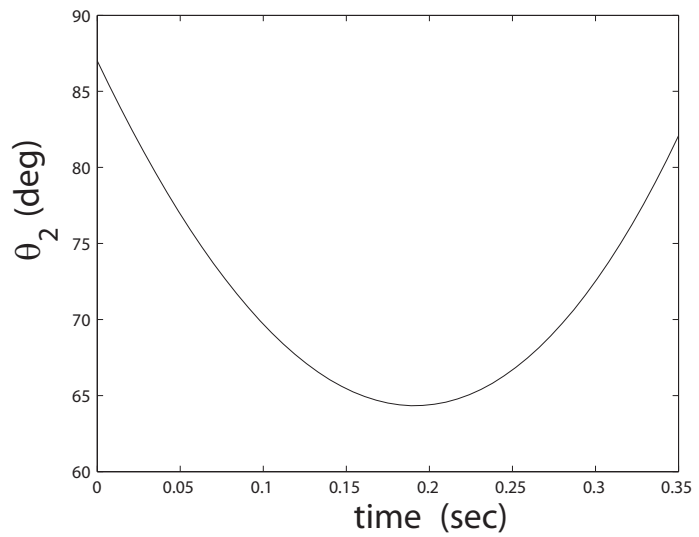

Fig. 13. Optimal trajectory for $\theta_{2}$ for the chosen step length

choosing a quadratic interpolation of $\theta_{1}$. The cubic interpolations of $\theta_{1}$ and $\theta_{2}$ from the optimal solution are shown in Fig. 12 and 13 respectively. Unlike the inverse dynamics case the angles do not decrease monotonically. $\theta_{1}$ first increases to $89^{\circ}$ and then decreases to $82^{\circ}$. This indicates a substantial difference in the behavior of the system. The optimal torque necessary for this motion is shown in Fig. 14. More extensive simulations are documented in Kappaganthu (2007).

\section{Experimental setup}

The aim of the experiment is to build a two dimensional biped that can be controlled with the torso. It is used to verify the controllability of the step length and velocity using a torso. The third dimension is neglected, steel guides are used to balance the biped in the 3rd dimension. Prismatic joints actuated by solenoids are used to provide sufficient clearance during the step take off. The solenoids are timed using the 555 timer circuit whose switches are placed at the 


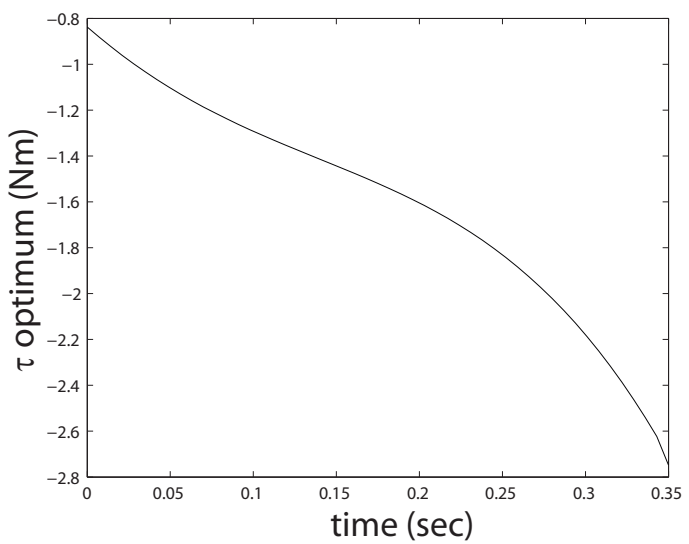

Fig. 14. Optimum torque obtained for the chosen step length

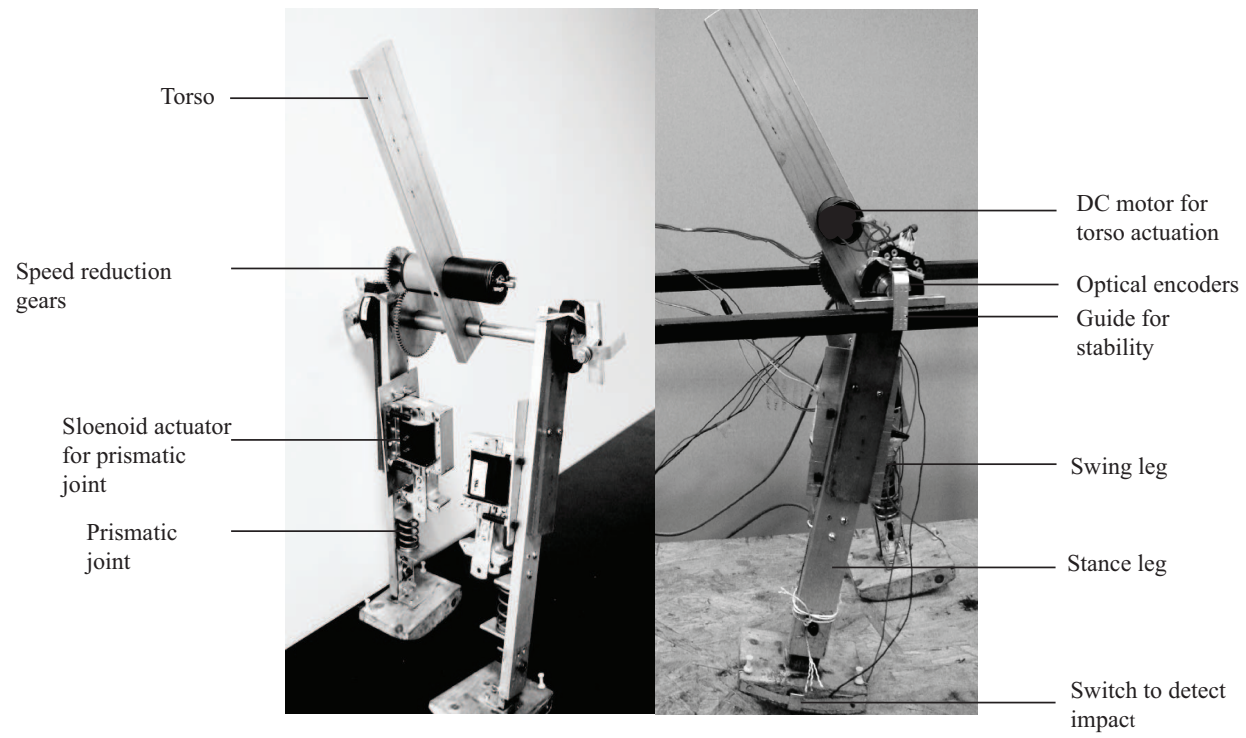

Fig. 15. The Villanova biped robot

feet. A motor is used to control the torso. dSpace is used to interface the hardware with the software. The biped robot built is shown in Fig. 15. The components of the biped and the principle of operation are explained in the following sections.

\subsection{Components}

\subsubsection{Legs}

Two aluminium $C$ channels .125 inches thick are used as legs. The length of the aluminum channels is 14 inches. Each leg is 1.5 inches wide, the length of the channelsŠ leg is .75 inches. A hole .25 inches in diameter is drilled at the top of each leg to connect the hip. A small hole 
.125 inches in diameter is drilled near the bottom to assemble the prismatic joint. Mounting bases, 5 inches long and 2.75 inches wide are provided at 3 inches from the top to mount the solenoids. An aluminium L angle support is attached at the 10 inches to load the spring of the prismatic joint.

\subsubsection{Prismatic joint}

A slider with a slot 1 inch long in a rectangular slab of $2.5 \times 1.26$ inches is attached to the leg with a screw. L angles of 1 inch are fixed at either end of the slider. The top angle is used to load a spring and the bottom angle is attached to the foot. A spring, 1.5 inch long, is placed between the angles on the leg and slider.

\subsubsection{Solenoid}

An AC intermittent solenoid is used to actuate the prismatic joint. The solenoid has a stroke length of $1 \mathrm{inch}$; it runs on 120VAC, $60 \mathrm{~Hz}$ current. The solenoid is mounted on the mounting base provided on the leg. The solenoid is 3 inches long and 2.33 inches wide. The pull force at 1 inch stroke length is $6 \mathrm{Lb}$. The solenoid weighs $2.7 \mathrm{Lb}$.

\subsubsection{Hip}

The hip made from a cylindrical aluminium rod of diameter .25 inches. The hip is 10 inches long. Three hex threads are machined at each end of the hip. Bearings 1 inch long made of PVC are attached at each end. The bearings are step turned to prevent play.

\subsubsection{Feet}

Feet are probably the most complicated parts to design. The feet design obtained after a lot of experimentation is 5 inches long and 3.25 inches wide and are carved from wood. Choosing the feet profile is a difficult task. It should be such that it does not interfere with the motion. As there is no actuation at the feet, it should be sufficiently curved to allow free rotation about the point of contact, however it should not be too steep as this would topple the biped. The profile has been obtained by trial and error. Each foot has a switch which turns on when the feet hit the slope.

\subsubsection{Torso, motor and gears}

The torso is made up of a 2 X14 inches aluminium block. A hole .25 inches in diameter is drilled at the end to connect it to the hip. The torso is fixed in place on the hip using cylindrical restraints. A DC servomotor is used to actuate the torso. The motor has an internal gear train for speed reduction. The motor has a nominal voltage of $24 \mathrm{VDC}$ and a stall torque of 1250 $\mathrm{mNm}$. It weighs 400 grams and is 3 inches long. The planetary gear train has a reduction ratio of 531:1. The maximum torque output in intermittent operation is $20 \mathrm{Nm}$. The length of motor gear train combination is 7 inches. The total weight is $1.5 \mathrm{Kg}$. Additionally a 1.75 inches diameter brass gear is attached to the shaft; this meshes with a gear 3 inches in diameter. The larger gear is fixed to the hip.

\subsubsection{Time circuit}

The 555 timer is used to switch the relay, which actuates the solenoid. The 555 timer is a circuit whose input is a trigger and the output is a step signal of known period. The period can be adjusted by changing the resistance and conductance in the circuit. A description of the circuit can be found in any popular book on circuits. The time period of the output pulse is given by 
Eq. 24, where $R_{v}$ and $C_{v}$ are the resistance and capacitance respectively.

$$
T_{\text {out }}=R_{v} C_{v}
$$

\subsubsection{Power sources and relays}

A total of four power sources are used. Two power sources are used to drive the motors in forward and backward direction. Agilent 3614A power sources are used. These can be remotely controlled using $\mathrm{dSpace}$ and have a gain of 20 and a range of $0-20 \mathrm{VDC}$. One power source is connected to the 555 timer circuit and the fourth powers the 12VDC relay which switches the solenoids. A total of six relays are used. Two 12VDC relays switch the solenoids. Four 7VDC SPDT relays are used to switch the two power sources controlling the forward and backward motion of the torso.

\subsubsection{Guideways}

When the solenoid is actuated the prismatic joint causes the feet to lift up; however because of the weight of leg the biped tilts sideways. In order to prevent this, guideways are used. These guideways support the biped at the hip. A better solution would be to use lighter actuators for the prismatic joint and provide a reactive force at the stance legs ankle. This has not been implemented as guideways are easier and cheaper to build and at the same time satisfy the requirement of validating the qualitative effect of the torso on walking in 2 dimensions. An ' $\mathrm{L}$ ' hook at the hip is used for safety to prevent the biped from deviating from the path and falling.

\subsection{Working principle}

There are three different processes happening at the same time, the up and down motion of the prismatic joints, the swinging of legs causing the biped to walk and the controlled motion of the torso. Data is collected from and transferred only in the latter two processes. The first process is autonomous to most extent. Fig. 17 and 16 explain the processes involved.

When the left leg hits the slope, the switch on it sends a signal to the first 555 timer circuit which sends a timed pulse to the solenoid attached to the right leg, this gives clearance to the leg, due to the dynamics of the system the leg moves forward and takes a step. The timer is set such that it is less than $50 \%$ of step period, this ensures that the leg gets back to its original length before the end of the step. When the right leg hits the slope the second 555 timer sends a signal to the left leg and the process repeats itself to create an obstructed motion.

The motor is actuated by an Agilent E3615A power source. The range of this power source is 0-20VDC; however, negative voltage needs to be applied to drive the motor in the reverse direction. This problem has been overcome by using two power sources. The power sources drive the motor in two opposite direction. This too has a problem; the power sources go into overdrive due to a short circuit between the independent power sources. To completely open the circuit when the power source is not in use, two relays are used. These relays are closed when the power source is to be inactive and open when the power source is to be active.

\section{Experimental results}

Data is collected and analyzed for three sets of data on a slope of $3^{\circ}$. In the first two cases the torso is fixed and no external torque is applied. The torso is fixed at two different positions, once leaning forward and once leaning backward. In the third case optimal torque is applied. 


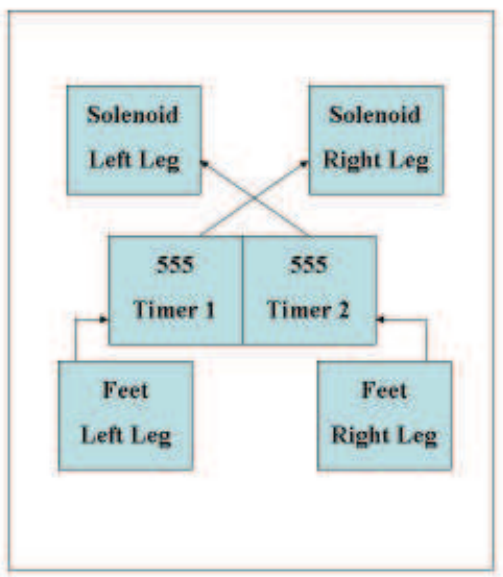

Fig. 16. Solenoid Control Schematic

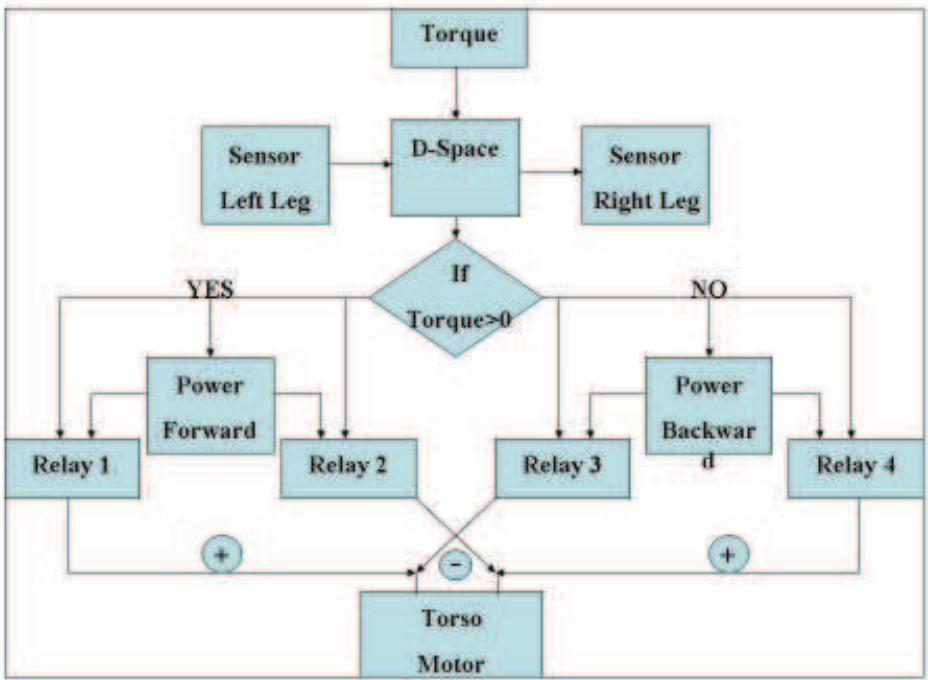

Fig. 17. Torso Control Schematic

\subsection{Fixed torso}

The profiles of $\theta_{1}$ and $\theta_{2}$ are obtained without a torque on the torso. In the first case the torso is held at an angle of $10^{\circ}$ and in the second case at an angle of $-10^{\circ}$. Fig. 18 show the raw data obtained with $\theta_{c}=10^{\circ}$ and Fig. 19 show the raw data obtained with $\theta_{\mathcal{c}}=-10^{\circ}$. It can 
be seen that there is no periodicity in the variation. Various initial conditions were tried but no limit cycle was found. Also it is not possible to wait for the biped to converge to a nearest limit cycle if it exists, since only 10 to 15 steps are possible on the ramp. The step profiles for a single step are shown in Figs. 20 and 21 for $\theta_{c}=10^{\circ}$ and $\theta_{\mathcal{c}}=-10^{\circ}$ respectively.

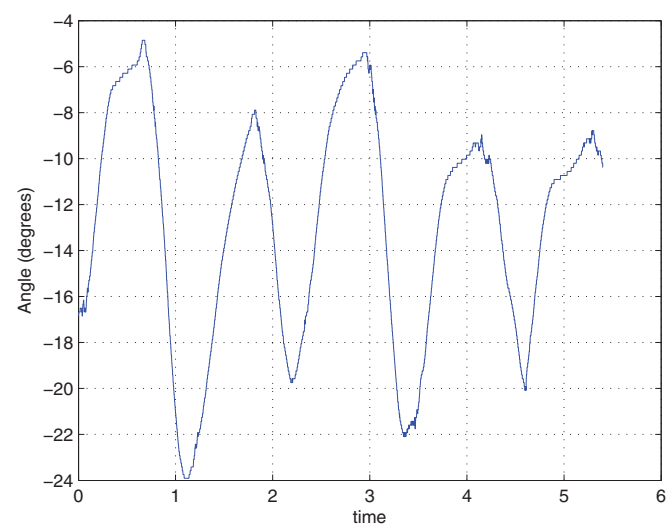

Fig. 18. Sensor output of angle subtended by the leg with vertical when $\theta_{C}=10^{\circ}$

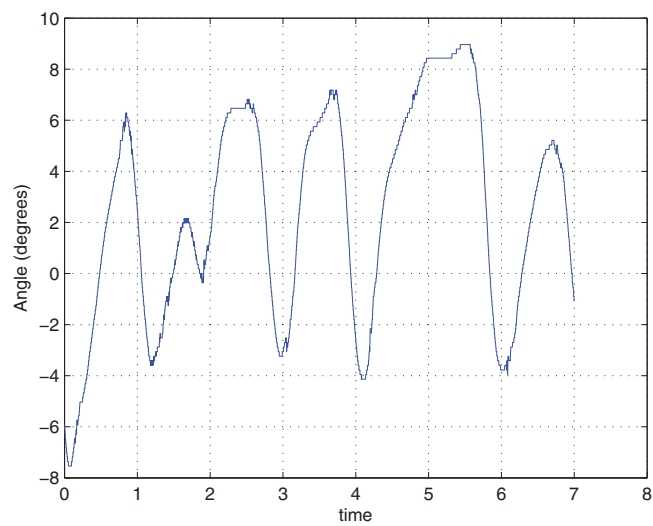

Fig. 19. Sensor output of angle subtended by the leg with vertical when $\theta_{C}=-10^{\circ}$

\subsection{Optimal torque}

The optimal torque is calculated for a step length of $6 \mathrm{~cm}$ and a time period of $.35 \mathrm{sec}$. However, again because of the the inherent differences between the mathematical model and the real system, there is a substantial difference between the time periods. The optimal torque obtained is stretched evenly over the real time period. After many iterations the torque's time period and the real time period were made to match at $.595 \mathrm{sec}$. The torque applied is shown in Fig. 22. Figure 23 show the raw data obtained from the sensor. This is more periodic and indicative of a useful walking robot. 


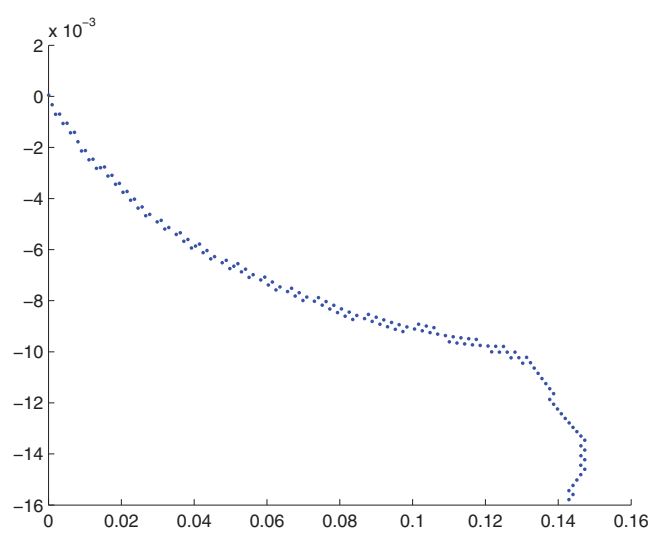

Fig. 20. Step profile when $\theta_{C}$ is held constant at $10^{\circ}$

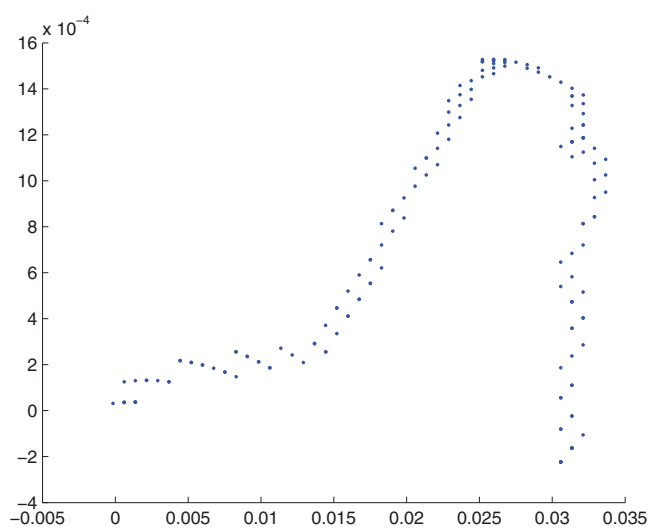

Fig. 21. Step profile when $\theta_{C}=10^{\circ}$

The comparison of experimental and numerical feet profiles is shown in Fig. 24. It can be seen that there is a marked qualitative similarity between the experimental and numerical results. Also it can be observed that there is more repetitiveness in this case when compared to the case with no torque. The experimental step length was $4.5 \mathrm{~cm}$.

\section{Conclusion}

This research has explored a novel aspect of biped robots with torsos. It has shown the importance and utility of the torso in the dynamics of the biped. The torso has been used efficiently to make the biped walk with the specified step length and velocity. The use of the torso has reduced the number of actuators required; further, the use of optimal torque has greatly reduced the external energy required for walking. The biped we analyzed, designed and constucted effectively uses the natural dynamics of the system; at the same time, the external excitation at torso has increased the practicability of the biped. 


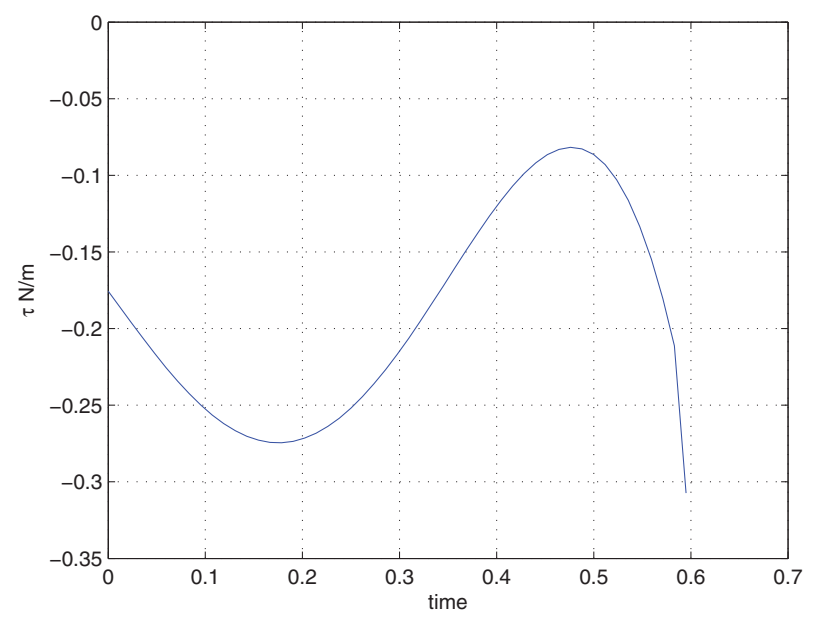

Fig. 22. Applied Optimal Torque

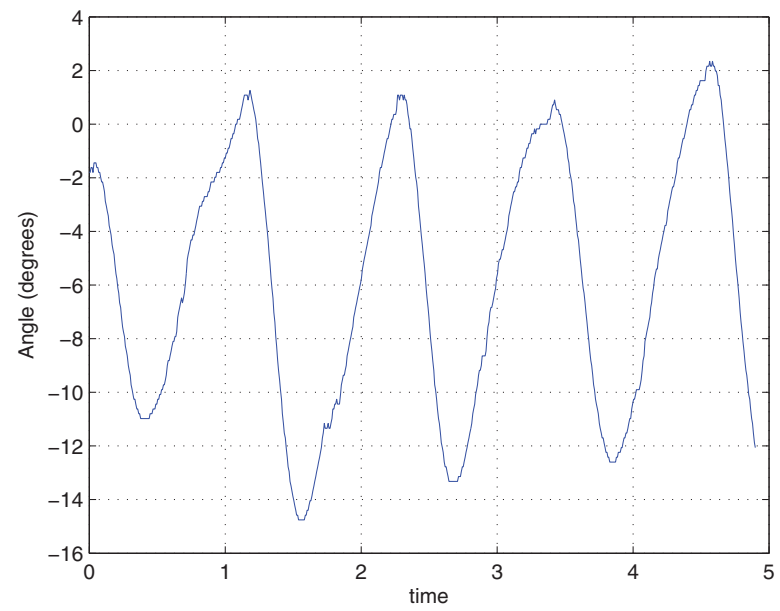

Fig. 23. Sensor output of the angle subtended by the leg with vertical when optimal torque is applied

A working model of the biped has been built. This biped has shown impressive qualitative agreement with numerical results. The Villanova biped walks on slopes, and the step-length and velocity can be controlled. When a torque acts on the biped it shows a higher repeatability of step-length than when no torque is applied. Optimal torque has been computed and applied, and the biped exhibits satisfactory performance.

Currently efforts are underway to add knees and feet to the analysis. Since the method is computationally expensive, work is being done to implement control using more efficient algorithms. 


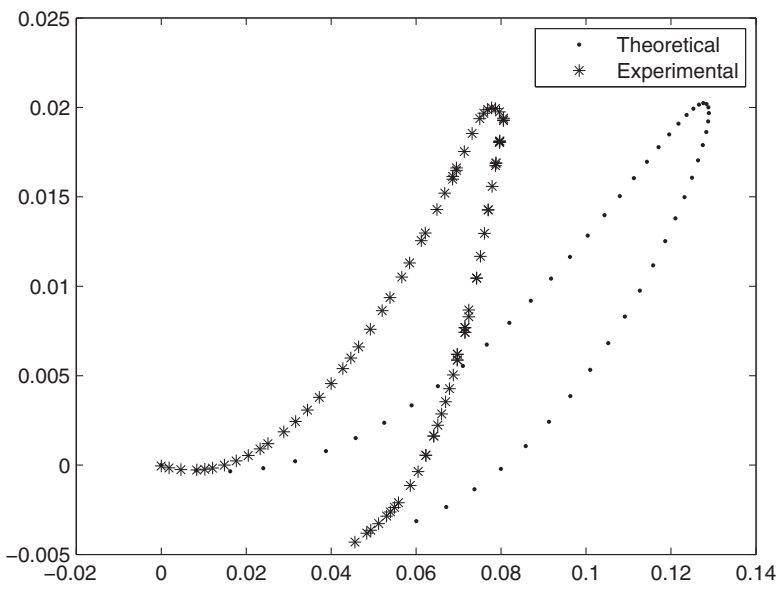

Fig. 24. Comparison of experimental and numerical step profiles when $\tau=\tau_{\text {optimal }}$

\section{References}

Asano, F., Luo, Z.-W. \& Yamakita, M. (2004). Some extensions of passive walking formula to active biped robots, Proceedings If 2004 IEEE, International Conference in Robotics and Automation pp. 3797-3802.

Asano, F., Yamakita, M., Kamamichi, N. \& Luo, Z.-W. (2004). A novel gait generation for biped walking robots based on mechanical energy constraint, IEEE Transactions on Robotics and Automation 20(3): 565-573.

Goswami, A. (1999). Postural stability of biped robots and the foot rotation Indicator(FRI) point, International Journal of Robotics Research .

Goswami, A., Espiau, B. \& Keramane, A. (1996). Limit cycles and their stability in a passive bipedal gait, IEEE International Conference onRobotics and Automation .

Goswami, A., Espiau, B. \& Keramane, A. (1997). Limit cycles in a passive compass gait biped and passivity mimicking control laws, International Journal of Robotics .

Goswami, A., Quaid, A. \& Peshkin, M. (1993). Complete parameter identification of a robot from partial pose information, IEEE International Conference on Robotics and Automation .

Goswami, A., Thuilot, B. \& Espiau, B. (1996). Compass-like biped robot part i: Stability and bifurcation of passive gaits, Technical report, Institut National De Recherche En Informatique Et En Autimatique.

Goswami, A., Thuilotz, B. \& Espiauy, B. (1996). A study of the passive gait of a compass like biped robot: Symmetry and chaos, International Journal of Robotics Research .

Kappaganthu, K. (2007). Design and development of a biped with a moving torso, Master's thesis, Villanova University.

Kim, J.-H. \& Oh, J.-H. (2004). Walking control of the humanoid platform KHR-1 based on torque feedback control, Proceedings of the 2004 IEEE, International Conference in Robotics and Automation pp. 622-625.

McGeer, T. (1990). Passive dynamic walking, International Journal of Robotics Research 9(2): 62-82. 
Paul, C., Yokoi, H. \& Matushita, K. (2003). Design and control of humanoid robot locomotion with passive legs and upper body actuation, International Symposium on Robotics .

Roussel, L., de Wit, C. C. \& Goswami, A. (98). Generation of energy optimal complete gait cycles for BipedRobots, IEEE International Conference on Robotics and Automation pp. 2036-2041.

Silva, F. M. \& Machado, J. A. T. (2001). Goal-oriented biped walking based on force interaction control, Proceedings of the 2001 IEEE, International Conference of Robotics and Automation pp. $4122-4127$. 


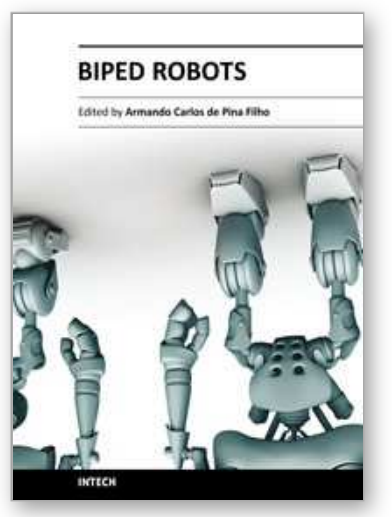

\author{
Biped Robots \\ Edited by Prof. Armando Carlos Pina Filho
}

ISBN 978-953-307-216-6

Hard cover, 322 pages

Publisher InTech

Published online 04, February, 2011

Published in print edition February, 2011

Biped robots represent a very interesting research subject, with several particularities and scope topics, such as: mechanical design, gait simulation, patterns generation, kinematics, dynamics, equilibrium, stability, kinds of control, adaptability, biomechanics, cybernetics, and rehabilitation technologies. We have diverse problems related to these topics, making the study of biped robots a very complex subject, and many times the results of researches are not totally satisfactory. However, with scientific and technological advances, based on theoretical and experimental works, many researchers have collaborated in the evolution of the biped robots design, looking for to develop autonomous systems, as well as to help in rehabilitation technologies of human beings. Thus, this book intends to present some works related to the study of biped robots, developed by researchers worldwide.

\title{
How to reference
}

In order to correctly reference this scholarly work, feel free to copy and paste the following:

Karthik Kappaganthu and C. Nataraj (2011). Optimal Biped Design Using a Moving Torso: Theory and Experiments, Biped Robots, Prof. Armando Carlos Pina Filho (Ed.), ISBN: 978-953-307-216-6, InTech, Available from: http://www.intechopen.com/books/biped-robots/optimal-biped-design-using-a-moving-torsotheory-and-experiments

\section{INTECH}

open science | open minds

\author{
InTech Europe \\ University Campus STeP Ri \\ Slavka Krautzeka 83/A \\ 51000 Rijeka, Croatia \\ Phone: +385 (51) 770447 \\ Fax: +385 (51) 686166 \\ www.intechopen.com
}

\author{
InTech China \\ Unit 405, Office Block, Hotel Equatorial Shanghai \\ No.65, Yan An Road (West), Shanghai, 200040, China \\ 中国上海市延安西路65号上海国际贵都大饭店办公楼 405 单元 \\ Phone: +86-21-62489820 \\ Fax: +86-21-62489821
}


(C) 2011 The Author(s). Licensee IntechOpen. This chapter is distributed under the terms of the Creative Commons Attribution-NonCommercialShareAlike-3.0 License, which permits use, distribution and reproduction for non-commercial purposes, provided the original is properly cited and derivative works building on this content are distributed under the same license. 